Orthopäde 2006 $35: 296-305$ DOI 10.1007/s00132-005-0921-7

Online publiziert: 24. Januar 2006

(c) Springer Medizin Verlag 2006

U. Weber · Y. Robinson · R. Kayser

Zentrum für spezielle Chirurgie des Bewegungsapparates, Klinik und Hochschulambulanz

für Orthopädie, Campus Benjamin Franklin, Charité - Universitätsmedizin Berlin

\title{
Seltene krankhafte
}

Veränderungen der oberen Halswirbelsäule mit operativer Behandlungsindikation

stellung des Kopfes mit Linksseitneigung und Rotationsfehlstellung sowie eine erhebliche Einengung des Spinalkanals.

Es wird eine zweizeitige operative Korrektur mit transoraler Lösung und transoraler Spinalkanaldekompression (Densresektion) und Ruhigstellung in Haloweste sowie eine definitive Stabilisierung durch dorsale Instrumentation Co-C6 durchgeführt.

Neben diesen weitgehend bekannten Veränderungen finden sich eine Reihe ungewöhnlicher pathologischer Erscheinungen der oberen HWS ebenfalls mit operativer Behandlungsnotwendigkeit. Auch wenn diese gelegentlich nicht isolierte Erkrankungen des okzipitozervikalen Übergangs darstellen, werden sie aber wegen der resultierenden biomechanischen Funktionsstörung und mehr noch wegen der drohenden neurologischen Komplikationen häufig zum Hauptsymptom einer ggf. zugrunde liegenden allgemeinen Erkrankung. Vor allem die ausgeprägte Gefahr schwerwiegender neurologischer Komplikationen rechtfertigt ihre detaillierte $\mathrm{Be}$ trachtung. Aufgrund der Seltenheit dieser Veränderungen lässt sich ihre Problematik am ehesten durch die Darstellung von Einzelcharakteristiken verdeutlichen.

\section{Neurofibromatose}

Die Neurofibromatose (• Abb. 2, 3) wird den sog. Phakomatosen zugeordnet. $\mathrm{Da}$ runter versteht man neurokutane Syn- drome, die durch Dysplasien insbesondere neuroektodermaler Gewebe charakterisiert sind und die zur Ausbildung ektodermaler oder mesenchymaler Tumoren sowie angeborener Gefäßveränderungen führen [22]. Die zugrunde liegenden Gendefekte sind inzwischen teilweise bekannt. Neben der Neurofibromatose werden zu den Phakomatosen u. a. das BournevillePringle-Syndrom, das Hippel-Lindau-Syndrom, die Sturge-Weber-Krabbe-Krankheit, das Peutz-Jeghers-Syndrom, der multiple Glomustumor u. a. gezählt.

Aufgrund klinischer und molekularbiologischer Kriterien wird bei der Neurofibromatose zwischen einem Typ I und Typ II unterschieden. Orthopädische Manifestationen finden sich ausschließlich bei einer Neurofibromatose Typ I (NF I). Die Neurofibromatose Typ II (auch zentrale Neurofibromatose genannt) ist wesentlich seltener als die NF I - klinisch ist sie durch das Auftreten meist bilateraler Tumoren des 8. Hirnnervs (sog. Akustikusneurinome) gekennzeichnet.

Die Neurofibromatose Typ I wird auch als periphere Neurofibromatose oder als Morbus Recklinghausen bezeichnet. Es handelt sich um eine autosomal dominant vererbte Erkrankung mit einer Häufigkeit von 1:40oo. Sie weist keine Geschlechtsunterschiede auf.

Die NF I stellt eine der häufigsten Erbkrankheiten dar. Genetisch handelt es sich um eine Mutation eines auf dem langen 

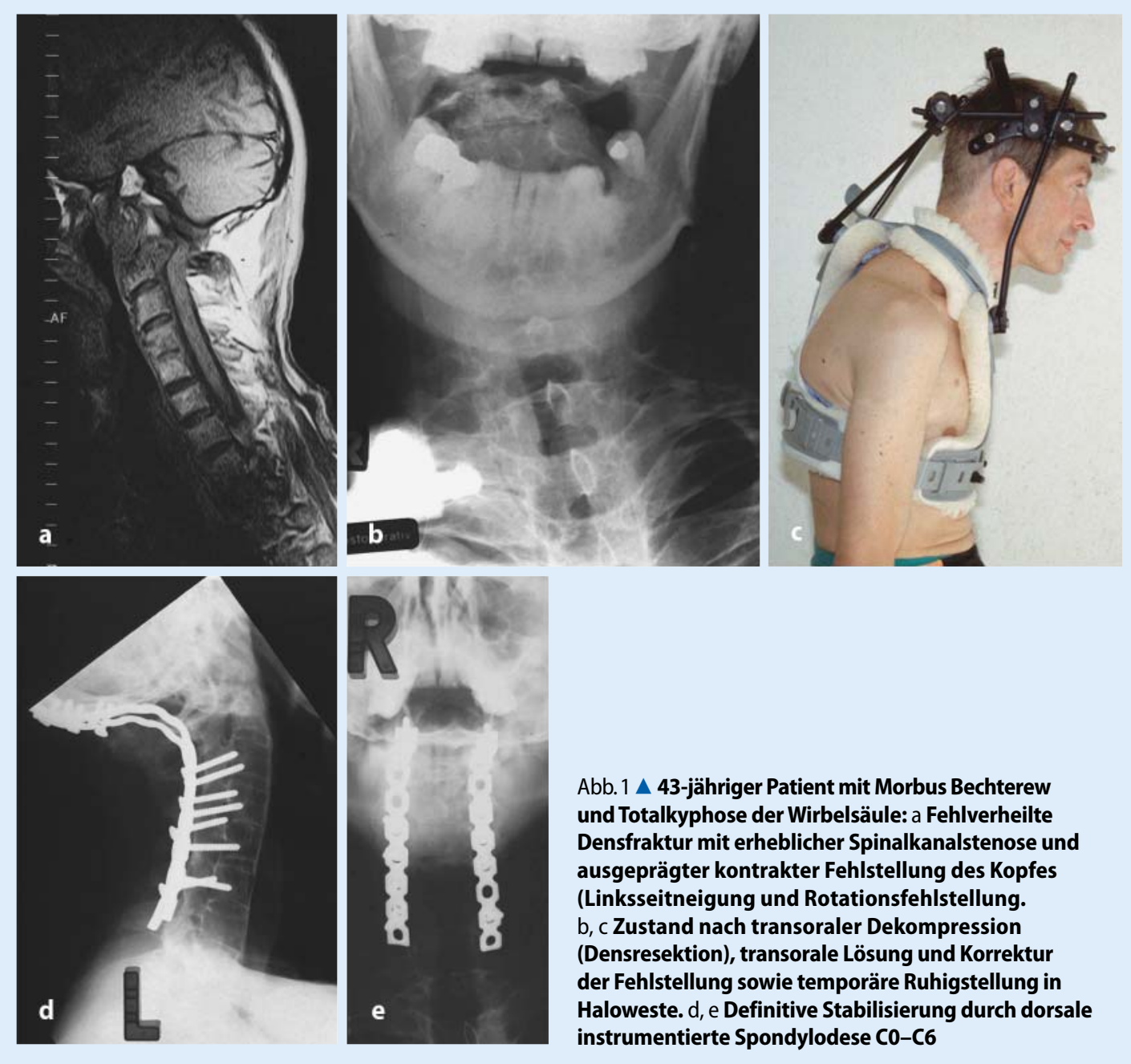

\begin{abstract}
Abb. $1 \Delta$ 43-jähriger Patient mit Morbus Bechterew und Totalkyphose der Wirbelsäule: a Fehlverheilte Densfraktur mit erheblicher Spinalkanalstenose und ausgeprägter kontrakter Fehlstellung des Kopfes (Linksseitneigung und Rotationsfehlstellung. b, c Zustand nach transoraler Dekompression (Densresektion), transorale Lösung und Korrektur der Fehlstellung sowie temporäre Ruhigstellung in Haloweste. d, e Definitive Stabilisierung durch dorsale instrumentierte Spondylodese C0-C6
\end{abstract}

Arm von Chromosom 17 (17Q11.2) lokalisierten Gen, welches die Expression von Neurofibromin, einem Tumorsuppressorprotein, kodiert. Durch den Verlust von Neurofibromin soll die Entstehung von gutartigen und bösartigen Tumoren bei NF I-Patienten ausgelöst werden. Da das Tumorsuppressorprotein in vielfältiger Weise die Zellproliferation und Zelldifferenzierung beeinflusst, ist der Verlust von Neurofibromin möglicherweise auch für zahlreiche andere klinische Manifestationen der Neurofibromatose verantwortlich. Welcher Anteil dem Neurofibrominmangel an der Entstehung der orthopädischen Manifestationen zukommt, ist derzeit unklar [14].

Klinisch ist die Neurofibromatose Typ I durch die typischen Hyperpigmentierungen (Café-au-lait-Flecken), durch sog. Lisch-Knötchen (Irishamartome), plexiforme Neurofibrome (tiefer gelegene Neurofibrome im Bereich größerer
Nervenstränge) und besonders auffällig durch die dermalen Neurofibrome charakterisiert. Bei den dermalen Fibromen handelt es sich um kleine, in großer Zahl an der Haut auftretende Neurofibrome an den Endaufzweigungen der Hautnerven. Bei der NF I sind daneben u. a. vielfältige Durchblutungsstörungen bekannt, die durch umschriebene zelluläre Hyperproliferationen in den Gefäßwänden zustande kommen - sie gelten als eine der wesentlichen Ursachen für häufig profuse Blutungen im Rahmen von orthopädischen Operationen bei Neurofibromatose, z. B. bei Skolioseoperationen.

Etwa die Hälfte aller Patienten mit NF I weist klinisch relevante Veränderungen im Bereich des Haltungs- und Bewegungsapparats auf [3]. Typische orthopädische Manifestationen der Neurofibromatose Typ I sind Skoliosen und Skoliokyphosen der Rumpfwirbelsäule, frühkindliche Verkrümmungen langer Röhrenknochen, die sog. angeborene Schienbeinpseudarthrose, Exkavationen von Wirbelkörpern (Scalloping) und eine diffuse Osteoporose. Etwa 20\% der Patienten mit Neurofibromatose Typ I entwickeln Wirbelsäulendeformierungen (Skoliosen/Kyphoskoliosen). Dabei machen die Skoliosen bei Neurofibromatosen im Kindes- und Jugendalter nur insgesamt etwa 1\% aller Skoliosen aus. Die Skoliosen bei Neurofibromatosen werden üblicherweise in sog. dystrophe und in nicht-dystrophe Formen unterschieden [1].

Die Ursache der Wirbelsäulenveränderungen, sowohl der Dystrophien (z. B. Exkavationen) wie der reinen Wirbelsäulendeformierungen (Skoliosen, Kyphoskoliosen) ist unverändert unklar; unstrittig ist, dass sich die Skoliosen bei der Neurofibromatose klinisch und prognostisch von anderen Skoliosen im Kindes- und Jugendalter unterscheiden. Für die dystrophen Skoliosen typisch sind kurzbogige Verkrümmungen 
Hier steht eine Anzeige.

黛 Springer 
Orthopäde 2006 · 35:296-305

DOI 10.1007/s00132-005-0921-7

(c) Springer Medizin Verlag 2006

\section{U. Weber · Y. Robinson · R. Kayser}

\section{Seltene krankhafte Veränderungen der oberen Halswirbelsäule mit operativer Behandlungsindikation}

\section{Zusammenfassung}

Die obere Halswirbelsäule (HWS) weist aufgrund ihrer anatomischen Besonderheiten gegenüber den übrigen Wirbelsäulenabschnitten andere, für diesen Bereich typische Erkrankungen und Verletzungsformen auf. Für die Mehrzahl der krankhaften Veränderungen des okzipitozervikalen Übergangs sind in den letzten 2 Jahrzehnten standardisierte Behandlungshierarchien definiert worden. Daneben finden sich typische, aber seltene krankhafte Veränderungen:

Etwa 20\% der Patienten mit Neurofibromatose I entwickeln Wirbelsäulendeformierungen. Diese spielen sich hauptsächlich im Bereich der Rumpfwirbelsäule ab (dystrophe/nicht-dystrophe Formen). Dystrophe Formen der Wirbelsäulendeformie- rung können in seltenen Fällen auch die HWS unter Einschluss der oberen HWS betreffen und zu grotesken Verbiegungen mit Kompromittierung des Spinalkanals führen. Wie bei den dystrophen Deformierungen im Bereich der Rumpfwirbelsäule ist eine aggressive, frühzeitige und kombinierte operative Therapie erforderlich.

Beim Morbus Down sollte eine konsequente und regelmäßige Mitbeurteilung der oberen HWS erfolgen. Auch wenn operationspflichtige Instabilitäten, $z$. B. ein instabiles Os odontoideum, vergleichsweise selten sind, muss den Veränderungen der oberen HWS beim Down-Syndrom besondere Beachtung geschenkt werden, da neurologische Auffälligkeiten aufgrund der Besonderheiten der Grunderkrankung häufig lange Zeit verkannt werden. Die operative Therapie des instabilen Os odontoideum beim Morbus-Down-Syndrom folgt den allgemeinen Regeln dieser pathoanatomischen Variation.

Auch beim Klippel-Feil-Syndrom sollten regelhaft seltene Begleitfehlbildungen im Bereich der oberen HWS durch geeignete diagnostische Verfahren ausgeschlossen werden.

\section{Schlüsselwörter}

Obere Halswirbelsäule .

Wirbelsäulendeformierungen .

Neurofibromatose .

Morbus-Down-Syndrom .

Klippel-Feil-Syndrom · Morbus Bechterew

\section{Rare pathological alterations of the upper cervical spine requiring surgical treatment}

\section{Abstract}

Because of its unique anatomy, specific diseases and lesions arise in the upper cervical spine, which differ widely from the rest of the spine. During the last two decades standardised diagnostic and therapeutic algorithms have been defined for most of the craniocervical pathologies often occurring in combination with an underlying disease requiring surgical intervention as well. On the other hand there are some very rare phathological alterations:

About $20 \%$ of the patients suffering from neurofibromatosis type I develop spinal deformities. These are mostly found in the thoracic and lumbar spine (dystrophic/ non-dystrophic type). In rare cases the dys- trophic neurofibromatosis type I involves the upper cervical spine leading to bizarre deformities endangering the spinal cord. An aggressive, timely and combined operative therapy is necessary.

Patients with Down syndrome should be investigated regularly for affections of the upper cervical spine. Though only in about $1 \%$ of all patients with Down syndrome do instabilities require surgical intervention, the upper cervical spine should be screened on a regular basis, since neurological changes due to the pathognomy of the underlying disease often remain undetected for a long time. The operative therapy of the instable os odontoideum in Down syndrome follows the general principles of this pathoanatomical variation.

Even though the Klippel-Feil syndrome is generally not linked with neuropathological findings, rare associated deformities of the upper cervical spine should be excluded by proper diagnostic procedures.

\section{Keywords}

Upper cervical spine.

Deformities of the spine .

Neurofibromatosis - Down syndrome .

Klippel-Feil syndrome .

Bechterew syndrome 

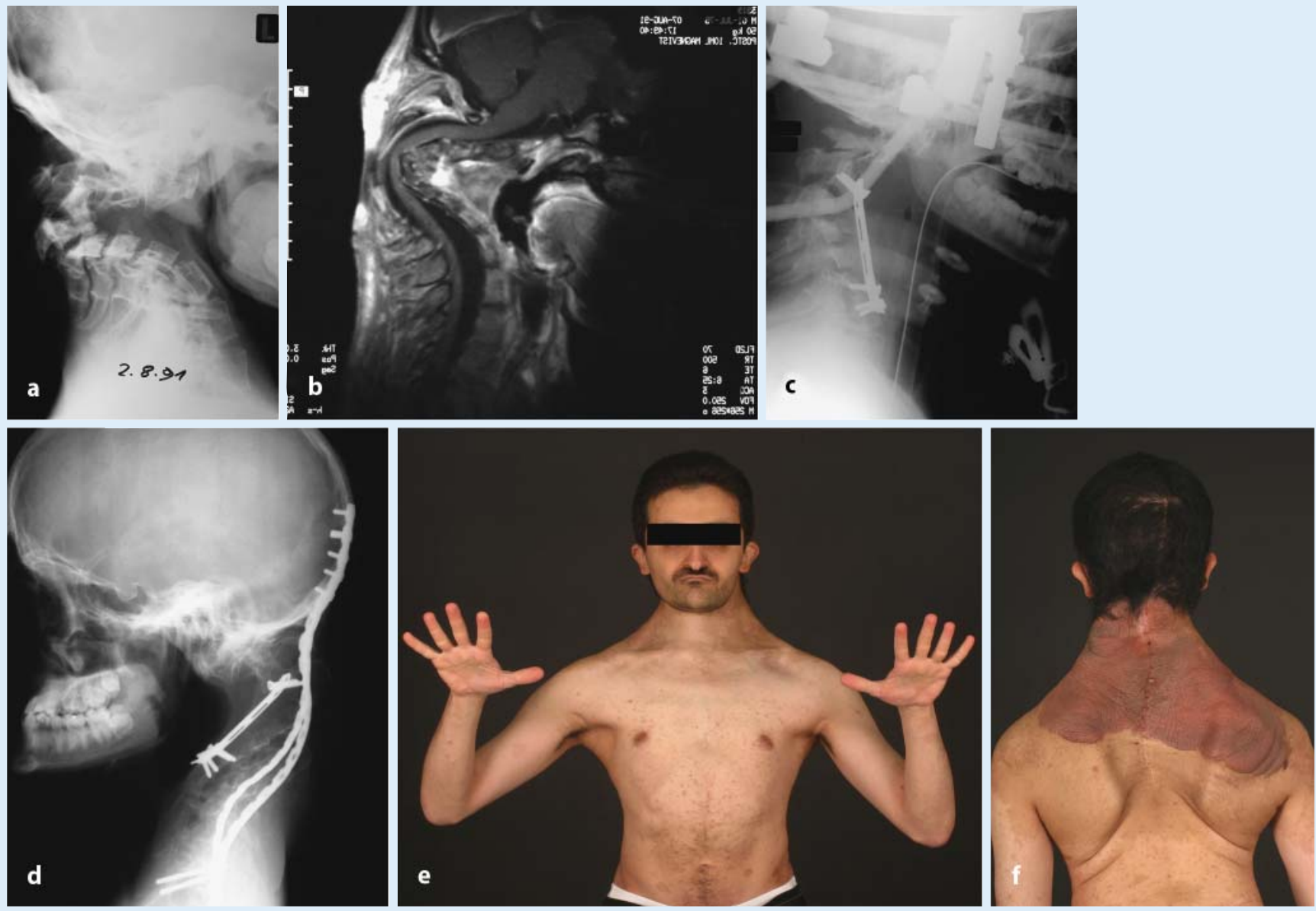

Abb. 2 × 17-jähriger Patient mit Neurofibromatose Typ I und dystropher Kyphoskoliose der HWS, Tetraparese. a, b Präoperativer Befund. c Zustand nach mehrwöchiger Distraktionskorrektur in Haloweste und Zustand nach ventraler Dekompression durch Mehretagenspondylektomie mit ventraler instrumentierter Spondylodese. $d$ Zustand nach dorsaler langstreckiger Stabilisierung $\mathrm{C} 0$-Th6. e, $\mathrm{f}$ Zustand 15 Jahre nach operativer Versorgung, zufrieden stellender klinischer Befund, keine neurologischen Auffälligkeiten
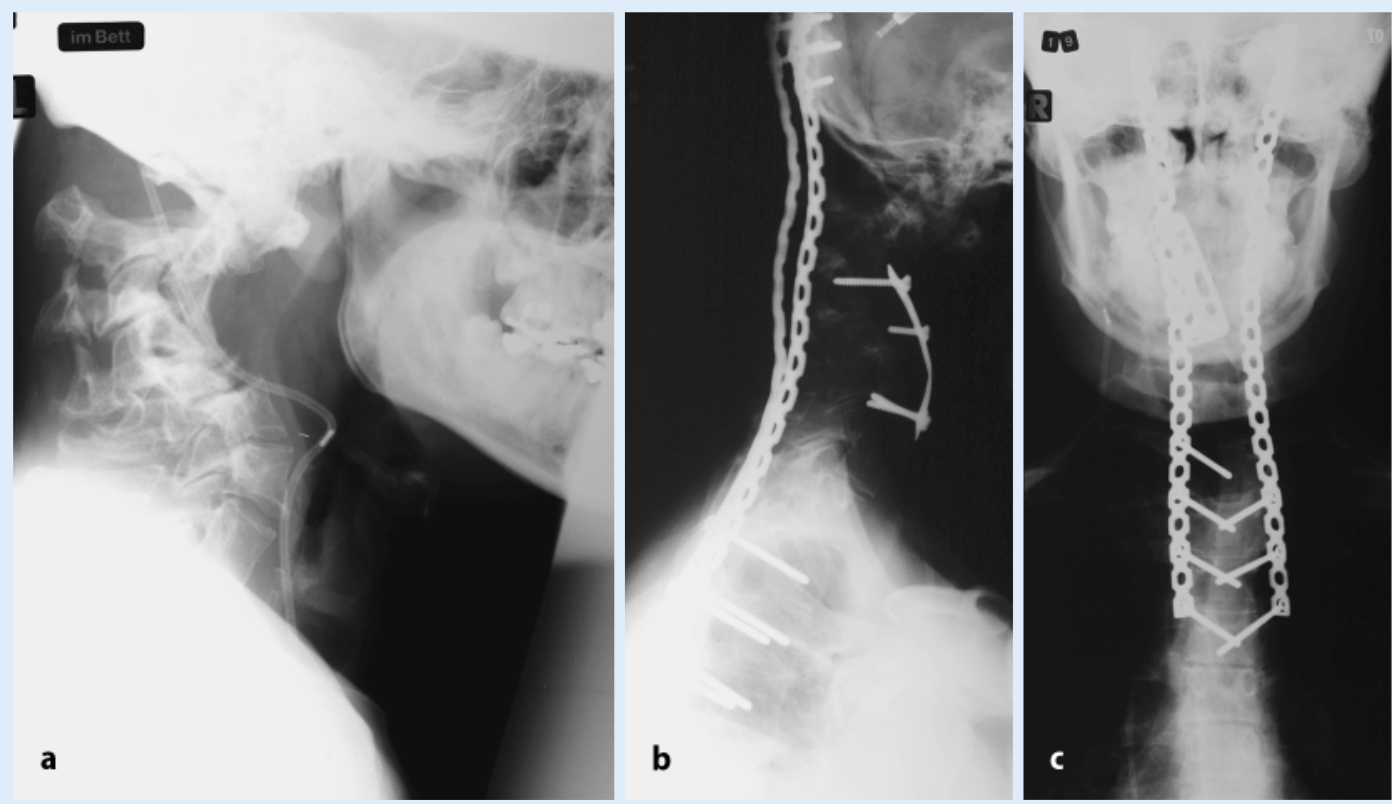

Abb.3 $<$ 32-jähriger Patient mit bekannter Neurofibromatose: Zustand nach ventrikuloatrialem Shunt wegen progredienter Tetraparese. a Ausgeprägte Kyphoskoliose der HWS mit hochgradiger Spinalkanalstenose und zervikaler Myelopathie. b, c Zustand nach zweizeitiger kombinierter ventrodorsaler operativer Versorgung, ventrale Dekompression mit Mehretagenspondylektomie und instrumentierter Spanspondylodese und (in 2. Sitzung) dorsale langstreckige Stabilisierung C0-Th5. Postoperativ vollständige Rückbildung der neurologischen Ausfälle 


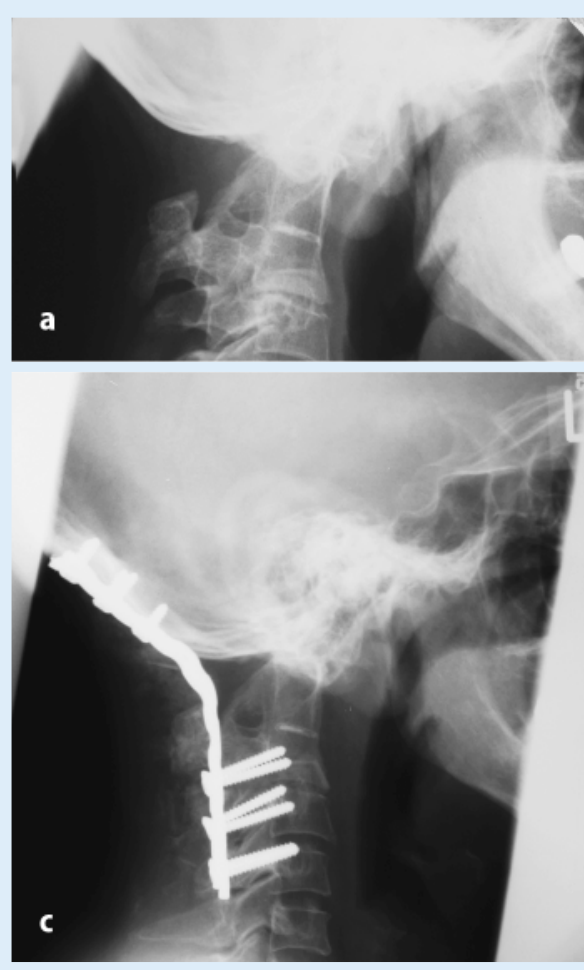

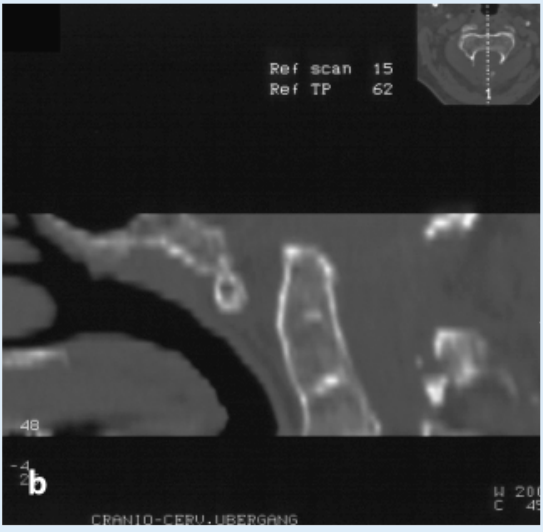

Abb. $4 \Delta$ 64-jährige Patientin mit KlippelFeil-Syndrom sowie basilärer Impression und zervikaler Myelopathie. Zustand nach auswärtiger dorsaler Operation mit Erweiterung des Foramen magnum und Bogenresektion C1. a Röntgenbefund mit verbliebener basilärer Impression. b CT-Befund (sagittale Rekonstruktion. c Zustand nach transoraler Dekompression mit subtotaler $\mathrm{C} 2$-Resektion und dorsale Stabilisierung CO nach C5. Postoperativ inkomplette Rückbildung der Tetraparese
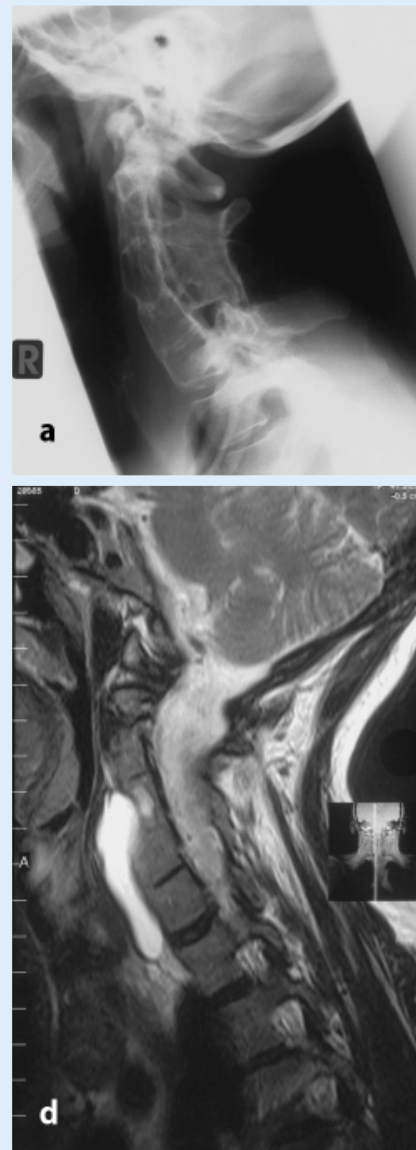
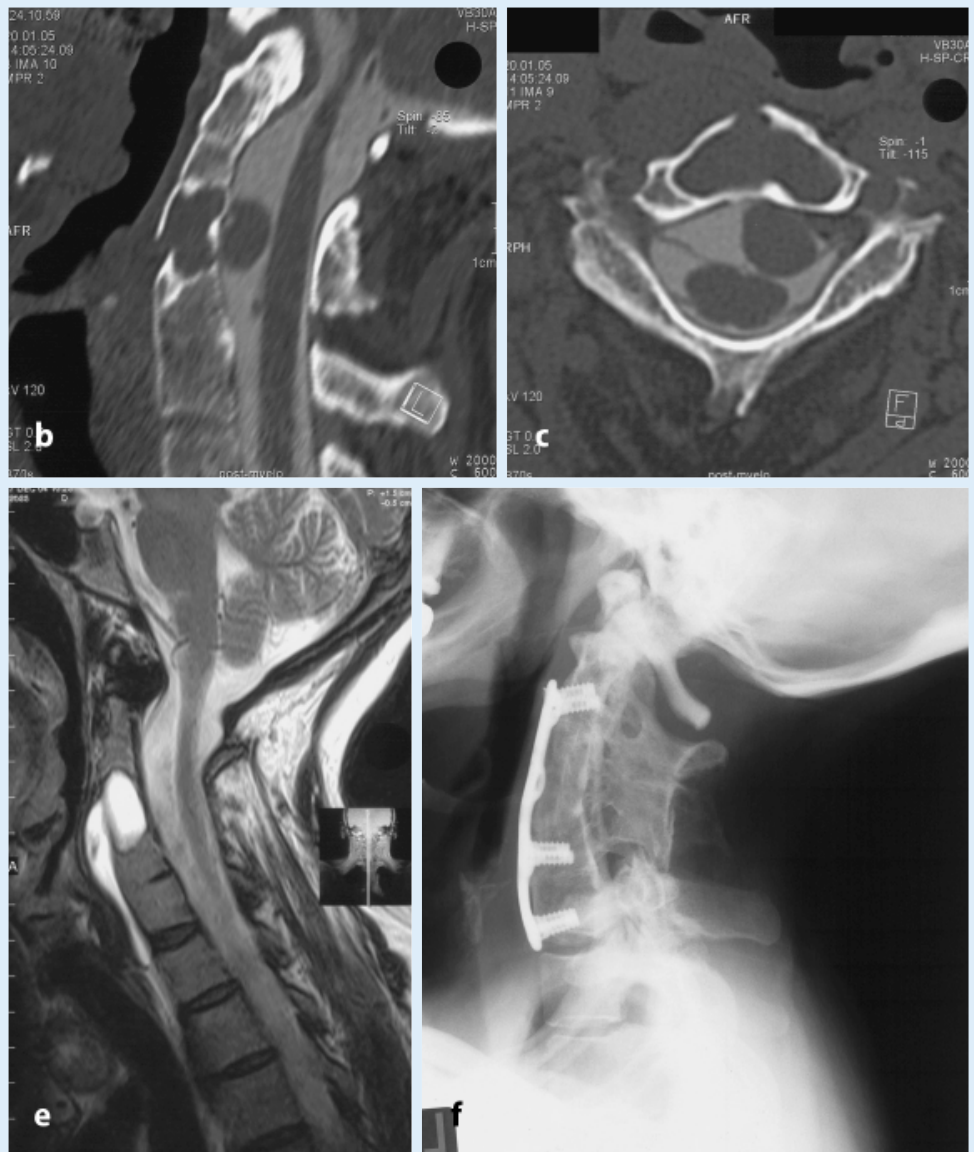

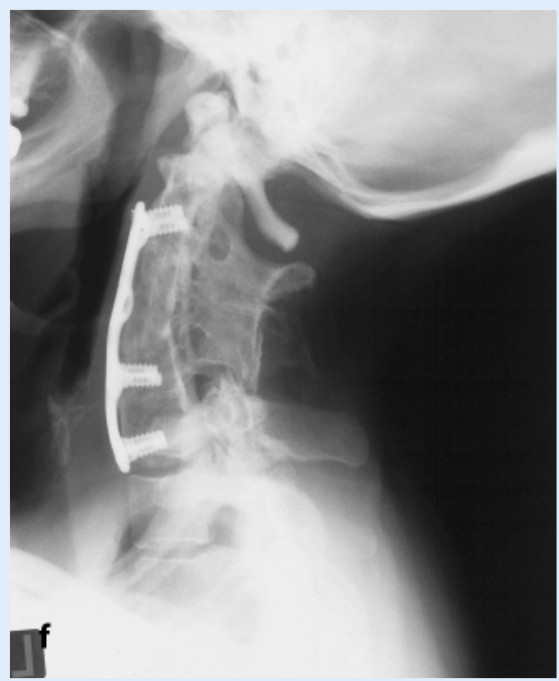

Abb. $5<45$-jährige Patientin mit KlippelFeil-Syndrom und enterogener Zyste: a Zystische Läsion C3. b Sagittale CT-Rekonstruktion; die ventrale knöcherne Defektbildung mit Verbindung zum prävertebralen Zystenanteil ist gut erkennbar. C CT-Darstellung der isolierten intraspinalen subarachnoidalen Zyste ohne Myelonkompression. $\mathrm{d}$, e MRT-Darstellung der prävertebralen Zyste. f Zustand 6 Monate nach operativer Versorgung (Zystenresektion, Stabilisierung) 


\section{Leitthema}
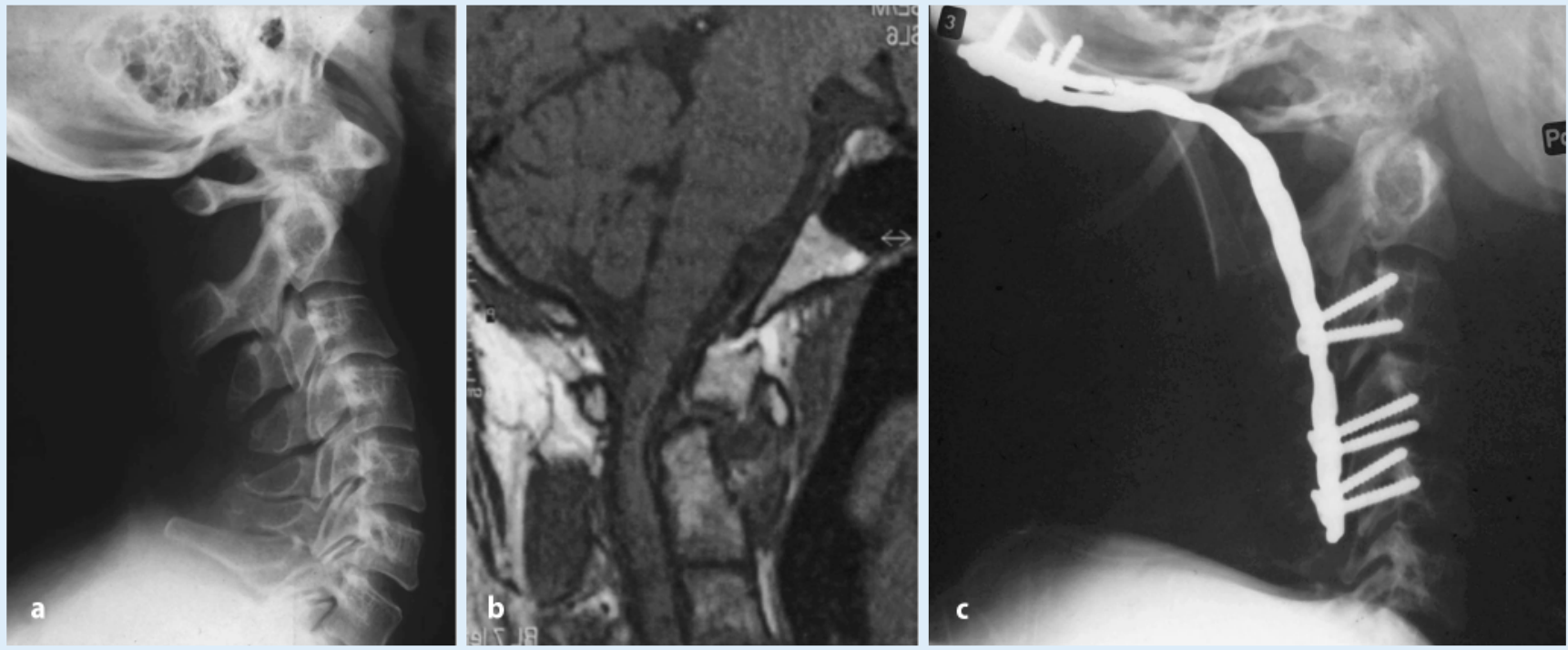

Abb. 6 \36-jähriger Patient mit disloziertem instabilem Os odontoideum: a Präoperativer Röntgenbefund. $b$ Im MRT deutliche Spinalkanalstenose. $c$ Zustand nach transoraler Dekompression mit Mobilisierung und Reposition der kontrakten HWS-Fehlstellung, Zustand nach dorsaler Stabilisierung C0 nach C5
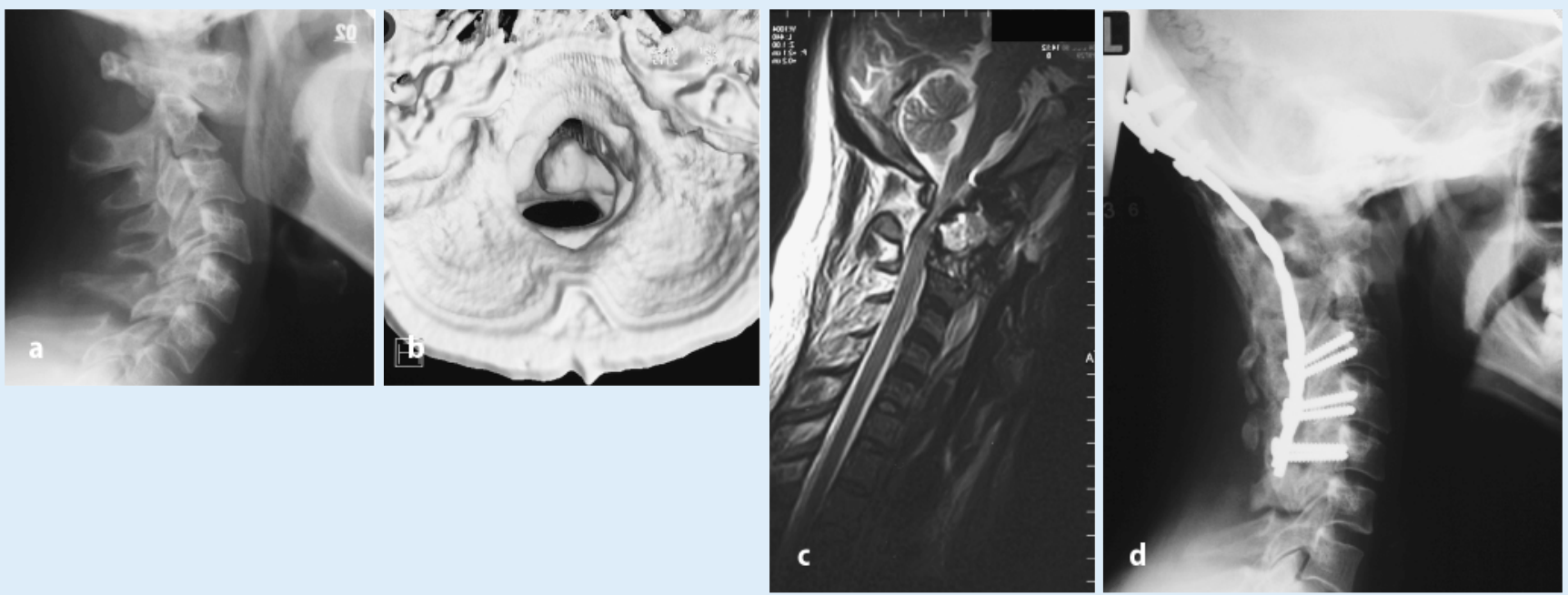

Abb.7 \ 34-jähriger Patient mit Down-Syndrom, ausgeprägte Tetraspastik: a, b Instabiles disloziertes Os odontoideum mit zervikaler Myelopathie, in der CT-3D-Rekonstruktion steht der Dens zentral im Spinalkanal. c MRT mit hochgradiger Spinalkanalstenose und zervikaler Myelopathie. d Zustand 1 Jahr nach zweizeitiger ventrodorsal kombinierter operativer Therapie, ventrale transorale Dekompression, dorsale Dekompression mit Resektion des hinteren Atlasbogens und Erweiterung des Foramen magnum, dorsale Stabilisierung $\mathrm{C} 0$ auf $\mathrm{C} 6$. Vollständige Rückbildung der neurologischen Symptomatik

im thorakalen Bereich, die frühzeitig klinisch manifest werden, mit einer ausgesprochenen Neigung zu neurologischen Störungen und mit rascher Progredienz.

Die sog. nicht-dystrophen Skoliosen sind eher den üblichen idiopathischen Skoliosen vergleichbar, auch wenn sie früher in Erscheinung treten und insgesamt eine schlechtere Prognose aufweisen. Darüber hinaus ist zu berücksichtigen, dass etwa die Hälfte aller primär nicht-dystrophen Formen später in dystrophe Formen übergehen soll [6]. Für die nicht-dystrophen Formen der Wirbelsäulendeformierungen gelten im Wesentlichen die für die übrigen Skoliosen im Kindes- und Jugendalter allgemein anerkannten Therapieprinzipien. Für die dystrophen Formen wird ein frühzeitiges aggressives Vorgehen, mit primär sehr ausgedehnten Versteifungsstrecken und ei- nem in der Regel ventrodorsal kombinierten Vorgehen empfohlen. Dabei ist die operative Behandlung von Skoliosen und $\mathrm{Ky}$ phoskoliosen der Rumpfwirbelsäule bei diesen Skoliosen mit einer hohen Komplikationsquote und teilweise sehr schwerwiegenden Komplikationen verbunden [12, 23].

Über die Beteiligung der HWS bei den muskuloskeletalen Manifestationen der Neurofibromatose sind nur wenig Mit- 

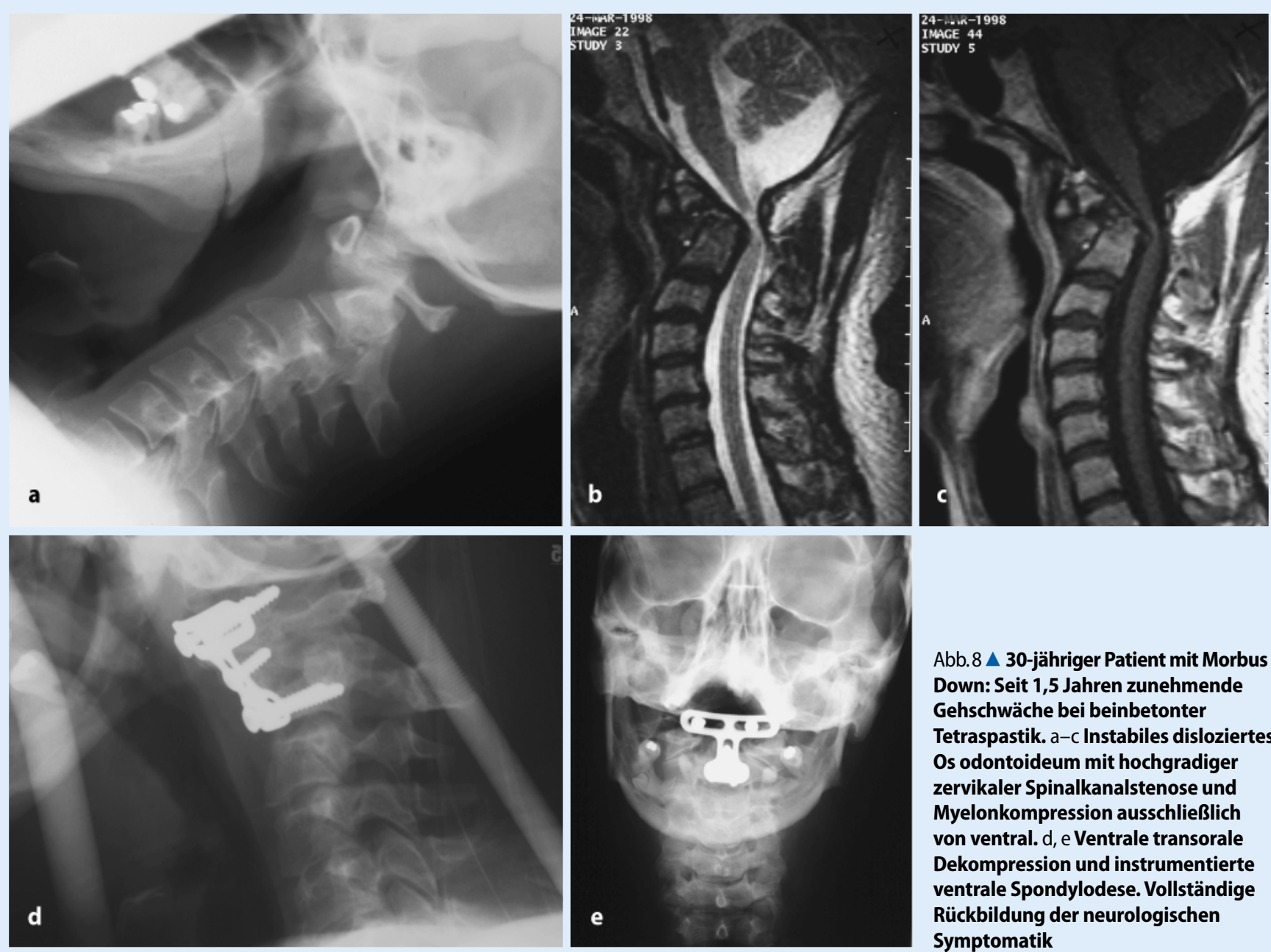

Abb. 8 \30-jähriger Patient mit Morbus Down: Seit 1,5 Jahren zunehmende Gehschwäche bei beinbetonter Tetraspastik. a-c Instabiles disloziertes Os odontoideum mit hochgradiger zervikaler Spinalkanalstenose und Myelonkompression ausschließlich von ventral. $d$, $e$ Ventrale transorale Dekompression und instrumentierte ventrale Spondylodese. Vollständige Rückbildung der neurologischen Symptomatik

teilungen bekannt $[4,24]$. Unterschiedliche Formen wie Neuroforamenerweiterungen, Hypolordosen, atlantoaxiale und atlantookzipitale Subluxationen, der Kollaps einzelner Wirbelkörper und fixierte Spondylolisthesen sind beschrieben. Darüber hinaus liegen Einzelmitteilungen über schwere, teilweise groteske Verbiegungen der HWS mit Beteiligung des okzipitozervikalen Übergangs im Sinne dystropher Wirbelsäulenveränderungen vor. Nach derzeitigem Kenntnisstand hat die Behandlung derartiger Deformierungen den Kriterien zu folgen, die für die dystrophen Wirbelsäulenveränderungen im Rumpfbereich Gültigkeit haben. Behandlungsziel ist die Herstellung eines dauerhaft stabilen Zustands, mit Teilkorrektur der Deformierung, Dekompression des Spinalkanals, Vermeidung neurologischer Komplikationen und Herstellung einer biomechanisch tolerablen Situation.

Im Fall 2 geht es um einen zum Zeitpunkt der Erstbehandlung 17-jährigen $\mathrm{Pa}$ - tienten (s. • Abb. 2), Neurofibromatose Typ I, mit grotesker Fehlform der HWS, zervikaler Myelopathie und beginnender Tetraparese. Nach Teilkorrektur durch Haloextension erfolgte eine zweizeitige kombinierte ventrodorsale operative Versorgung: in der 1. Sitzung eine ventrale Dekompression durch Mehretagenspondylektomie und ventrale instrumentierte Spanspondylodese $\mathrm{C}_{2}$ nach $\mathrm{C}_{7}$, in der 2. Sitzung eine dorsale langstreckige Stabilisierung Co nach Th6. 15 Jahre nach der operativen Versorgung ist der Befund klinisch zufriedenstellend, es zeigen sich keine neurologischen Auffälligkeiten (s. - Abb. 2).

\section{Klippel-Feil-Syndrom}

Beim Klippel-Feil-Syndrom handelt es sich um eine 1912 von Klippel u. Feil erstmalig beschriebene Veränderung im Bereich der HWS [13]. Sie ist durch eine vollständige oder unvollständige Verschmel- zung mehrerer oder aller Halswirbel gekennzeichnet. Die Gesamtzahl der Halswirbel kann dabei vermindert sein $[7,19]$.

Es handelt sich um eine Entwicklungsstörung (Segmentationsstörung) mit autosomal dominantem Erbgang. Röntgenologisch und kernspintomografisch finden sich Blockwirbelbildungen in unterschiedlichem Ausmaß. Degenerative Veränderungen der verbliebenen Bandscheibenlogen sind vermehrt in den darüber und darunter liegenden Etagen nachweisbar.

Die Fehlbildung ist häufig mit weiteren Veränderungen kombiniert: es können sich Halsrippen, lipomatöse und angiomatöse Tumoren in der Nackenregion, Rippenfehlbildungen, kongenitale Skoliosen und Kyphosen, Syringomyelien, Enzephalozelen, faziale und kraniale Asymmetrien, Sprengel-Deformitäten u. a. in 2040\% ausbilden. Als begleitende Veränderungen des okzipitozervikalen Übergangs sind basiläre Impressionen/basiläre Invaginationen beschrieben. 
In Fall 3 wird ein Klippel-Feil-Syndrom mit basilärer Impression und zervikaler Myelopathie dargestellt (• Abb. 4): Zustand nach auswärtiger dorsaler Voroperation mit Erweiterung des Foramen magnum und Bogenresektion $\mathrm{C} 1$, ohne Besserung der neurologischen Symptomatik. Transorale Dekompression $\mathrm{C}_{2} / \mathrm{C}_{3}$ und Densresektion sowie dorsale Stabilisierung Co nach $\mathrm{C}_{5}$.

\section{Enterogene Zysten}

Enterogene Zysten im Bereich der HWS werden als Fehlformationen, die aus dem Endoderm entstehen, angesehen. Möglicherweise sind sie das Ergebnis einer fehlerhaften Separierung der Chorda dorsalis von der Kopfdarmanlage, mit persistierenden endodermalen Anteilen in der Wirbelsäulenformation. Dabei kann eine persistierende Verbindung zwischen Kopfdarm und enterogener Zyste erhalten bleiben oder eine isolierte Zyste (z. B. intradural) resultieren. Enterogene Zysten sind gut abgegrenzte, flüssigkeitsgefüllte Gebilde mit einer sehr dünnen Wand aus fibrösem Bindegewebe und einem einschichtigen Epithel [2, 16, 21]. Enterogene Zysten finden sich in aller Regel bei jüngeren Patienten an der Brustwirbelsäule oder HWS, häufig mit Wirbelanomalien vergesellschaftet (4050\%). Die Zysten sind fast ausnahmslos vor dem Rückenmark lokalisiert. Die klinischen Symptome sind unterschiedlich und von der Lokalisation der Zysten abhängig; Rückenmarkskompressionssyndrome sind häufig, septische Komplikationen können auftreten.

Ein Klippel-Feil-Syndrom mit enterogener Zyste wird in Fall 4 dargestellt (- Abb. 5): hier handelt es sich um intraossäre Zystenanteile $\left(\mathrm{C}_{3}\right)$ mit drohender pathologischer Fraktur, prävertebral ausgedehnten zystischen Veränderungen und isolierter intraspinaler, subarachnoidaler Zyste ohne Myelonkompression. Die operative Versorgung erfolgt durch Zystenentfernung und instrumentierte Spanstabilisierung.

\section{Os odontoideum}

Der Dens axis und der Wirbelkörper des 2. Halswirbels entwickeln sich aus un- terschiedlichen Anlagen. Bereits bei Abschluss der Verknorpelung stellen Wirbelkörper und Dens ein einheitliches Gebilde dar; allerdings erfolgt auch die Ossifikation für beide Anteile getrennt. Dabei besitzt der Dens drei unterschiedliche Ossifikationszentren. Aufgrund der komplizierten Entwicklungsvorgänge am 2. Halswirbel sind Fehlbildungen und Varianten in diesem Bereich häufig.

Von zahlreichen Autoren wird das Os odontoideum der Gruppe der Densfehlbildungen zugerechnet:

- Os odontoideum,

- Densaplasie,

- Aplasie der Densspitze,

- Ossiculum terminale

Dabei wird das Os odontoideum als Folge einer ausgebliebenen knöchernen Verschmelzung zwischen Densanlage und Wirbelkörper, mit verbleibender fibröser Brücke angesehen.

Andere Autoren halten das Os odontoideum für eine erworbene Veränderung. Die klinische Relevanz des Os odontoideum wird übereinstimmend darin gesehen, dass spontan oder in Folge eines Trauma eine Instabilität oder Dislokation mit Gefährdung des Myelons resultieren kann [5, $8,9,10,20]$.

Im Fall 5 geht es um einen 36-jährigen Patienten mit ausgeprägter Funktionsstörung und kontrakter Fehlhaltung der HWS. Ein HWS-Trauma in der Vorgeschichte war nicht erinnerlich: disloziertes instabiles Os odontoideum, transorale Dekompression, Mobilisierung und Reposition, dorsale Stabilisierung Co nach $\mathrm{C}_{5}$ (• Abb. 6).

\section{Trisomie 21}

Das durch die Trisomie 21 hervorgerufene klinische Bild des Down-Syndrom (- Abb. 7, 8) wurde bereits Mitte des 19. Jahrhunderts (1866, L. Down) beschrieben. Die zugrunde liegende numerische Aberration wurde 1959 nachgewiesen (Lejeune und Turpie). Genetisch wird zwischen einer sog. freien Trisomie (95\% der Patienten), einer kompletten Translokationstrisomie und einer partiellen Trisomie unterschieden. Pathogenetisch ist die 3 fache Dosis mehrerer Gene einer bestimmten Chro- mosomenregion für die charakteristische Merkmalskombination des Krankheitsbildes entscheidend. Im Ergebnis führt dies zu einer intra- und extrauterinen Fehlentwicklung fast sämtlicher Strukturen und Organe, mit Fehlbildungen und einer Tendenz zu vorzeitigen Alterungsprozessen.

Die Klinik des Langdon-Down-Syndrom ist gut bekannt und durch individuell unterschiedliche geistige Retardierung, typische Dysmorphie mit Minderwuchs, Brachy- und Mikrozephalgie, typische Physiognomie, Muskelhypotonie, Hand- und Fußdeformitäten und begleitende Herzfehler gekennzeichnet. Die Inzidenz beträgt 1:70o und ist mit dem $\mathrm{Al}$ ter der Mutter hoch korreliert [14]. Aufgrund einer allgemeinen Bindegewebeschwäche werden z. B. Fehlentwicklungen im Bereich des Kniegelenks (permanente Patellaluxationen) sowie auch Veränderungen im Bereich der oberen HWS beobachtet.

Seit der Erstbeschreibung von okzipitozervikalen Instabilitäten beim DownSyndrom durch Spitzer (1961) sind eine Reihe von Publikationen zu dieser Problematik erschienen. Leichtere atlantoaxiale Instabilitäten in Folge der mit der Krankheit verbundenen allgemeinen Bindegewebsschwäche sind häufig und werden bei etwa $15-40 \%$ aller Patienten angenommen $[11,15]$. Dabei soll die Inzidenz symptomatischer Instabilitäten allerdings $<1 \%$ betragen; insofern die Frage, ob und wann atlantookzipitale und atlantoaxiale Instabilitäten beim DownSyndrom operativ behandelt werden sollten, immer noch nicht einheitlich beantwortet wird.

Eine Besonderheit stellt in diesem $\mathrm{Zu}$ sammenhang das Os odontoideum dar (- Abb. 7 und 8). Es soll in 1-5\% aller Patienten mit Down-Syndrom auftreten $[17,18]$. Die spezielle Problematik des Os odontoideum beim Down-Syndrom ergibt sich offensichtlich daraus, dass langsam zunehmende, neurologische Symptome vom Patienten nicht ausreichend artikuliert und vom Umfeld des Patienten lange Zeit als Teil der Grunderkrankung und nicht als zusätzliches wirbelsäulenverursachtes Problem mit progredienten neurologischen Ausfällen wahrgenommen werden. Im eigenen Krankengut wurden 3 Patienten innerhalb von 5 Jahren mit 
Down-Syndrom, instabilem Os odontoideum und progredienter Neurologie mit nahezu gleichartiger Krankengeschichte und eindrucksvoll verzögerter Primärdiagnose beobachtet.

Fall 6 zeigt einen 34-jährigen Patienten mit Down-Syndrom (s. • Abb. 7): seit 2 Jahren, im Anschluss eine rechtsseitige Patellaluxation, zunehmende Verschlechterung des Gangbildes, die bei unterschiedlichen Facharztvorstellungen der Grunderkrankung und den Veränderungen im Bereich der peripheren Haltungs- und Bewegungsorgane (Patellaluxation) angedient wurde. Klinisch und neurophysiologisch rechts- und beinbetonte Tetraspastik bei zervikaler Myelopathie. Röntgenologisch instabiles disloziertes Os odontoideum mit Spinalkanalstenose und zervikaler Myelopathie. Transorale Dekompression, temporäre Fixierung in Haloweste, in 2. Sitzung dorsale Dekompression mit hinterer Atlasbogenresektion und Foramenerweiterung, dorsale Stabilisierung Co auf $\mathrm{C}_{5}$.

\section{Fazit für die Praxis}

Neben den bekannten typischen Verletzungen und krankhaften Veränderungen der HWS z. B. bei chronischer Polyarthritis, für die inzwischen standardisierte Therapiestrategien definiert werden konnten, gibt es weitere, seltene operationspflichtige Veränderungen in diesem Bereich. Sie stellen häufig ein, in der klinischen Symptomatik meist führendes, Symptom einer Grunderkrankung dar. Die klinische Problematik ergibt sich vorzugsweise aus der drohenden neurologischen Komplikation. Die Prognose ist damit insbesondere von einer frühzeitigen Diagnose und einer rechtzeitigen und adäquaten Therapie abhängig. Die dystrophen Formen der Neurofibromatose Typ I können in seltenen Fällen auch an der HWS manifest werden und zu teilweise grotesken Deformierungen führen. Wie bei den häufigeren Skoliosen/ Kyphoskoliosen im Rumpfbereich ist hier eine frühzeitige aggressive operative Behandlung indiziert.

Beim Morbus Down sollten wie bei der chronischen Polyarthritis regelmäßige Mitbeurteilungen der oberen HWS erfolgen, da eine Mitbeteiligung der oberen HWS mit atlantoaxialer Instabilität bei bis zu $40 \%$ der Patienten angenommen wird. Auch wenn die Anzahl der operationspflichtigen Instabilitäten mit 1\% der Patienten relativ klein zu sein scheint, ist gerade bei diesen Patienten besondere Aufmerksamkeit geboten, da Fehlinterpretationen neu auftretender neurologischer Symptome zu Lasten der Grunderkrankung häufig zu sein scheinen.

Auch beim Klippel-Feil-Syndrom muss auf begleitende seltene, ggf. das Myelon kompromittierende, Fehlbildungen wie basiläre Invagination oder enterogene Zysten geachtet werden.

\section{Korrespondierender Autor \\ Prof. Dr. U. Weber}

Zentrum für spezielle Chirurgie des Bewegungsapparates, Klinik und Hochschulambulanz für Orthopädie, Campus Benjamin Franklin, Charité, Universitätsmedizin, Hindenburgdamm 30, 12200 Berlin E-Mail: ulrich.weber@charite.de

Interessenkonflikt: Es besteht kein Interessenkonflikt. Der korrespondierende Autor versichert, dass keine Verbindungen mit einer Firma, deren Produkt in dem Artikel genannt ist, oder einer Firma, die ein Konkurrenzprodukt vertreibt, bestehen. Die Präsentation des Themas ist unabhängig und die Darstellung der Inhalte produktneutral.

\section{Literatur}

1. Betz RR, lorio R, Lombardi AV, Clancy M, Steel HH (1989) Scoliosis surgery in neurofibromatosis. Clin Orthop 245: 53-56

2. Brooks BS, Dural ER, El Gammal T et al (1993) Neuroimaging features of neurenteric cysts: analysis of nine cases and review of the literature. AJNR 14: 735-746

3. Crawford AH (1994) Neurofibromatosis. In: Weinstein SL (ed) The pediatric spine. Raven Press, New York, pp 619-649

4. Craig JB, Govender S (1992) Neurofibromatosis of the cervical spine. A report of eight cases. J Bone Joint Surg Br 74: 575-578

5. Crockard A, Stevens JM (1995) Craniovertebral junction anomalies in inherited disorders: part of the syndrome or caused by the disorder? Eur J Pediatr 154: 504-512

6. Durrani A, Crawford AH, Choudhry SN, Saifuddin A, Morley TR (2000) Modulation of spinal deformities in patients with neurofibromatosis type I. Spine 25: 69-75

7. Exner $G$ (1957) Variationen und Fehlbildungen der Wirbelsäule. In: Hohmann G, Hackenbroch M, Lindemann K (Hrsg) Handbuch der Orthopädie, Bd. II. Thieme, Stuttgart, S 51-98

8. Fielding JW, Hensinger RN, Hawkins RJ (1980) Os odontoideum. J Bone Joint Surg Am 62: 376-383
9. Forlin E, Herscovici D, Bowen JR (1992) Understanding the Os odontoideum. Orthop Rev 21: 14411447

10. Hellinger J (1982) Os odontoideum - Missbildung oder veraltete Defektpseudarthrose? Beitr Orthop Traumatol 29: 525-529

11. Hungerford GD, Akkaraju V, Rawe SE, Young GF (1981) Atlanto-occipital and atlanto-axial dislocations with spinal cord compression in Down's syndrome: a case report and review of the literature. Br J Radiol 54: 758-761

12. Kim HW, Weinstein SL (1997) Spine update. The management of scoliosis in neurofibromatosis. Spine 22: $2770-2776$

13. Klippel M, Feil A (1912) Un cas d'absence de vertébres cervicales. Nouv Iconogr Salpêt 25: 223

14. Lentze MJ, Schaub J, Schulte FJ, Spranger J (2003) Pädiatrie. Grundlagen und Praxis. Springer, Berlin Heidelberg New York Tokio

15. Menezes AH, Ryken TC (1992) Craniovertebral Abnormities in Down's Syndrome. Pediatr Neurosurg 18: $24-33$

16. Osborn AG (1994) Diagnostik Neuroradiology. Mosby, St. Louis

17. Schmidt C, Kittel J (1988) Die atlanto-axiale Dislokation bei Down-Syndrom und Os odontoideum. Fallbericht und Literaturüberblick. Psychiatr Neurol Med Psychol (Leipzig) 40(8): 480

18. Taggard DA, Menezes AH, Ryken TC (2000) Treatment of Down syndrome-associated craniovertebral junction abnormalities. J Neurosurg (Spine 2) 93: 205-213

19. Töndury G (1958) Entwicklungsgeschichte und Fehlbildungen der Wirbelsäule. Hippokrates, Stuttgart

20. Torklus D v, Gehle W (1968) Schwierigkeiten der Differentialdiagnose von Denspseudarthrose und Os odontoideum. In: Erdmann H (Hrsg) Die Begutachtung der verletzten Wirbelsäule. Hippokrates, Stuttgart

21. Ulmer JH, Elster AD, Ginsberg LE, Williams DW III (1993) Klippel-Feil-Syndrom: CT and MR of acquired and congenital abnormalities of the cervical spine and congenital abnormalities of the cervical spine and cord. J Comp Asst Tomogr 17: 215-224

22. Weber U, Müller K (1983) Weichteiltumoren. Thieme, Stuttgart New York

23. Winter RB, Moe JH, Bradford DS, Lonstein JE, Pedras CV, Weber AH (1979) Spinal deformity in neurofibromatosis. J Bone Joint Surg Am 61: 677-694

24. Yong-Hing K, Kalamchi A, MacEwen GD (1979) Cervical spine abnormalities in neurofibromatosis. J Bone Joint Surg Am 61: 695-699 